\title{
Sostenibilidad de sistemas agrícolas de fincas ecológicas y tradicionales en Costa Rica
}

\author{
Greivin Fallas ${ }^{1}$, Mario Chacon ${ }^{2}$ y Jonathan Castro ${ }^{3}$ \\ 'Fundación INCIENSA, Liberia, Guanacaste-Costa Rica; jipifallas@yahoo.com \\ ${ }^{2}$ Consultor Servicios de los Ecosistemas y Mitigación al Cambio Climático, San José-Costa Rica; mchacon@catie.ac.cr \\ ${ }^{3}$ Corporación Educativa para el Desarrollo Costarricense, Goicoechea, San José-Costa Rica; jonathan@cedeco.or.cr
}

Recibido 4-VIII-2009 Corregido 19-VIII-2009 Aceptado 22-IX-2009

\begin{abstract}
Sustainability of agricultural systems on ecological and conventional farms in Costa Rica. This research developed and validated a methodology to analyze sustainability in 17 farms located in seven Costa Rican municipalities. Farm size varied between 1,40 hectares and 17,2 hectares; the land was in use in ecological and conventional cultivation systems in coffee, sugar cane, vegetables, and cattle ranching. The methodology was developed by characterization of management systems and evaluation of sustainability indicators. In order to characterize the management system, the information compiled between 2004 and 2007 was analyzed in five major aspects: human, social, natural, economic, and physical. The sustainability analysis allowed 15 indicators to be organized under the attributes of: stability, adaptability, productivity, efficiency, participation, organization, and ecosystem services. Only the ecological farms are sustainable. We recommend this method for use in other tropical countries.
\end{abstract}

\section{KEY WORDS}

Sustainable development, sustainability indicators, ecological agriculture, conventional agriculture.

\section{RESUMEN}

Se desarrolló y validó una metodología para el análisis de la sostenibilidad en fincas con manejo ecológico y tradicional en diferentes regiones de Costa Rica, para esto se eligieron 17 fincas, cuyo tamaño osciló entre las 1,40 ha y 17,2 ha. Los sistemas de producción agrícola analizados en las fincas fueron café, caña de azúcar, hortalizas y ganadería (de carne). Se hizo una caracterización del sistema de manejo, selección y valoración de indicadores de sostenibilidad. La caracterización del sistema productivo fue por análisis de capitales social, económico, físico, humano y natural. Se seleccionaron 15 indicadores de sostenibilidad los cuales fueron agrupados en atributos de estabilidad, adaptabilidad, productividad, eficiencia, participación, organización comunal y servicios de ecosistemas. Las fincas con manejo ecológico tienden a la sostenibilidad productiva y ambiental en mayor grado que las fincas con manejo tradicional. Esta metodología demostró ser una herramienta sencilla y se recomienda su utilización para mejorar actividades productivas en los países tropicales.

\section{PALABRAS CLAVE}

Desarrollo sostenible, indicadores de sostenibilidad, agricultura ecológica, agricultura tradicional.
Los ecosistemas son de suma importancia para el ser humano dado que aportan una serie de servicios y recursos. Estos servicios y recursos los podemos clasificar en servicios de suministro (como alimentos, leña, fibra y recursos genéticos), de regulación (del clima, de enfermedades, de agua o polinización) y culturales (religión, agro ecoturismo, educativos y patrimonio cultural). El ser humano se ha servido de estos servicios de los ecosistemas para vivir, sin embargo, en los últimos 50 años la humanidad ha modificado los ecosistemas con mayor rapidez y amplitud que en ningún otro momento en su historia. Si bien esta modificación ha generado ganancias económicas y desarrollo, esto ha sido a expensas de la degradación de los ecosistemas naturales (WRI 2005).

Esta modificación de los ecosistemas esta muy ligada a la agricultura y sus prácticas de manejo tradicional, así se ha reconocido mundialmente y queda registrado en los más recientes informes presentados por la Organización de las Naciones Unidas para la Agricultura y la Alimentación (FAO 2007) y por el Panel Intergubernamental sobre Cambio Climático (IPCC 2007). Problemas como la deforestación, la contaminación de aguas por insumos químicos, la 
pérdida de biodiversidad, la degradación de suelos y el cambio climático, este último, debido al aumento en las concentraciones de gases de efecto invernadero (GEI), son catalogados en los informes como los más importantes provocados por la agricultura tradicional.

Dichos informes hacen un llamado para buscar prontas soluciones a la degradación de los ecosistemas e implementar estrategias para mitigar el cambio climático. Una de estas opciones es cambiar el manejo tradicional de los sistemas agrícolas por un manejo con enfoque ecológico, la cual se rige por principios ecológicos como la manutención o incremento de la diversidad, el uso no entrópico de los recursos naturales y la no utilización de abonos sintéticos e insumos químicos (Altieri \& Nicholls 2000).

En estudios realizados a sistemas ecológicos para determinar su aporte a la sostenibilidad de las comunidades (Astier et al. 1997, Altieri \& Nicholls 2000, Masera \& López 2000, Astier et al. 2004, Gomero \& Velásquez 2004, Cárdenas et al. 2007), evidencian la eficiencia de los agroecosistemas con el medio y un manejo más sostenible. Esta sostenibilidad o desarrollo sostenible implica un proceso que debe ser estable y regenerativo en el tiempo, con una visión más amplia de lo que conlleva el desarrollo económico y la conservación de los recursos naturales. Este término de sostenible es más alternativo y defendido por diferentes autores (Leff, 1996 \& 2001, Bifani 1995, Mooney 1993) manifestando que lo sostenible complementa la ecología, economía y lo social, es decir se produce garantizando el estado natural de los ecosistemas mediante la distribución equitativa entre los distintos grupos sociales.

El objetivo de la presente investigación fue analizar 17 fincas, de las cuales 10 aplican manejo ecológico y 7 aplican manejo tradicional, distribuidas en siete cantones de Costa Rica, mediante indicadores sociales, económicos y ambientales. Se buscó con esto aportar nuevos conocimientos e indicadores sobre el manejo de fincas mediante el uso de técnicas en agricultura ecológica, además de generar una metodología que permita evaluar fincas ecológicas desde un punto de vista más sencillo y a bajo costo. Se espera que la información generada por este estudio, motive a la comunidad científica a seguir evaluando la agricultura ecológica y se espera además que influya a los tomadores de decisión en la creación de políticas ambientales que resuelvan los problemas nacionales e internacionales.

\section{METODOLOGÍA}

\section{Zona de estudio}

Las fincas seleccionadas se ubican en los cantones de Alfaro Ruiz, San Ramón (provincia de Alajuela); Aserrí, Acosta (provincia de San José); y Guácimo, Pococí y Siquirres (provincia de Limón). En total se analizaron 17 fincas (Fig. 1), las que se caracterizan en el Cuadro 1.

Las fincas ecológicas (a excepción de dos fincas) se denominaron de esta manera, debido a que se encuentran certificadas por la agencia certificadora costarricense Eco-LOGICA S.A. y BCS Öko Garantie, lo cual tiene como objetivo garantizar que sistemas de producción ecológica cumplan con la agricultura sostenible y buenas prácticas agrícolas. Y las fincas tradicionales se denominan aquellas en las cuales el manejo del sistema esta basado en el monocultivo y empleo de insumos agrícolas, así como, no se encuentran certificadas en agricultura ecológica.

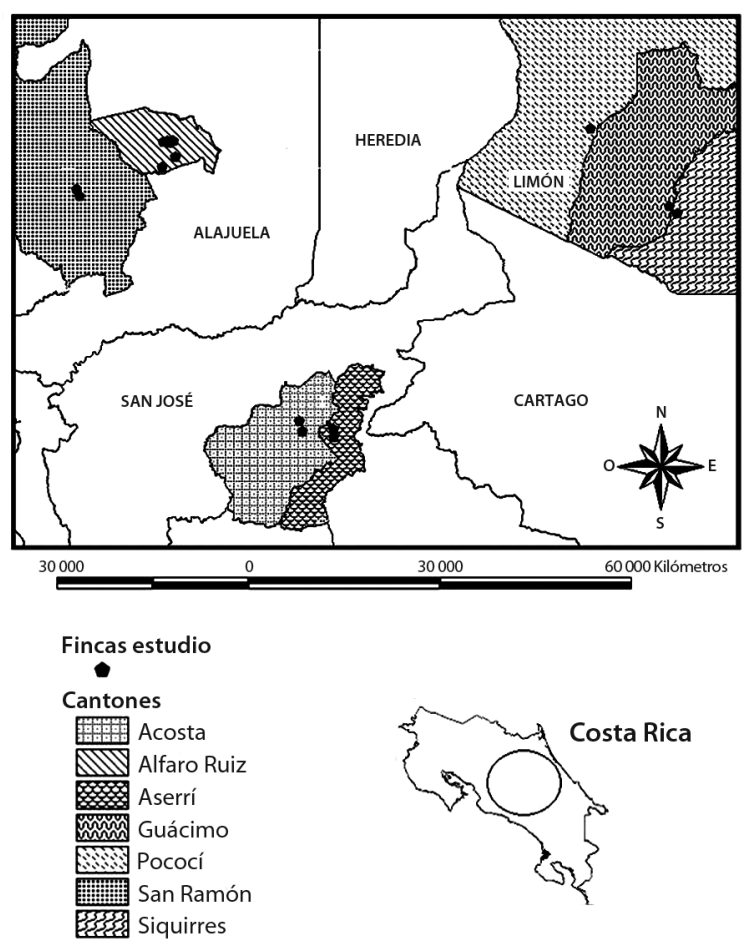

FIG. 1. Ubicación de las 17 fincas que formaron parte de la investigación, por cantón. 
CUADRO 1

Características principales de las fincas a analizar según tipo de sistema agrícola

\begin{tabular}{|c|c|c|c|c|c|c|c|}
\hline $\begin{array}{l}\text { Tipo de sistema } \\
\text { agrícola }\end{array}$ & Finca & $\begin{array}{l}\text { Certificada } \\
\text { por }\end{array}$ & $\begin{array}{l}\text { Zona de } \\
\text { vida }\end{array}$ & Cantón & $\begin{array}{c}\text { Temperatura } \\
\text { media }\left({ }^{\circ} \mathrm{C}\right)\end{array}$ & $\begin{array}{l}\text { Altura } \\
\text { (msnm) }\end{array}$ & $\begin{array}{c}\text { Precipitación } \\
\text { anual }(\mathrm{mm})\end{array}$ \\
\hline \multirow[t]{10}{*}{ Ecológico } & La Loma & Eco. Lógica S.A. & Bmh-TP & Acosta & 23 & 1000 & 3000 \\
\hline & El Guerrero & BCS Öko Garantie & Bmh-MB & Alfaro Ruiz & 17 & 1800 & 2500 \\
\hline & $J \& B$ & BCS Öko Garantie & Bmh-MB & Alfaro Ruiz & 17 & 1800 & 2500 \\
\hline & Guadalupe & BCS Öko Garantie & Bmh-MB & Alfaro Ruiz & 16 & 1800 & 2500 \\
\hline & Los Bobos & Eco. Lógica S.A. & Bmh-P & Aserrí & 23 & 1200 & 2500 \\
\hline & Marcos Vindas & Eco. Lógica S.A. & Bmh-P & Aserrí & 25 & 800 & 2200 \\
\hline & LTAV & Eco. Lógica S.A. & Bmh-P & Pococí & 20 & 1100 & 3500 \\
\hline & Jesús Maria & Eco. Lógica S.A. & Bmh-P & San Ramón & 20 & 1100 & 3500 \\
\hline & La Amistad & En transición & Bmh-P & Siquirres & 24 & 500 & 4000 \\
\hline & Jaime Chávez & En transición & Bmh-MB & Alfaro Ruiz & 16 & 1800 & 2500 \\
\hline \multirow[t]{7}{*}{ Tradicional } & Delfín Alfaro & No certificada & Bmh-MB & Alfaro Ruiz & 16 & 1800 & 2500 \\
\hline & La Esperanza & No certificada & Bmh-MB & Alfaro Ruiz & 16 & 1960 & 2500 \\
\hline & Hnos Rodriguez & No certificada & Bmh-MB & Alfaro Ruiz & 16 & 1800 & 2500 \\
\hline & Bernardo Naranjo & No certificada & Bmh-P & Aserrí & 24 & 1100 & 2500 \\
\hline & Olman Díaz & No certificada & Bmh-P & Aserrí & 23 & 900 & 1900 \\
\hline & Rancho Alegre & No certificada & Bmh-TB & Guácimo & 23 & 300 & 4000 \\
\hline & La Palma & No certificada & Bmh-P & San Ramón & 20 & 1100 & 3500 \\
\hline
\end{tabular}

\section{Caracterización de las fincas}

La información utilizada para la caracterización de las fincas, se originó de las bases de datos proporcionadas por la Corporación Educativa para el Desarrollo Costarricense (CEDECO) mediante el proyecto Emisión de Gases con Efecto Invernadero y Agricultura Orgánica desarrollado durante 2004 al 2007. La metodología empleada por CEDECO se encuentra en la publicación Enfoque metodológico: emisión de gases efecto invernadero, la fijación de carbono y la agricultura orgánica (Castro \& Amador 2006).

CEDECO, fue fundada en 1984, a partir del esfuerzo unificado de un grupo de personas con conocimientos en ciencias sociales y naturales, entre ellos agrónomos, forestales, sociólogos y educadores con experiencia en trabajo comunal y campesino. El objetivo de CEDECO es apoyar la agricultura ecológica como opción alternativa y afirmativa del nuevo modelo de desarrollo rural.

La caracterización de las fincas se llevó a cabo por

medio de cinco capitales: humano, social, natural, físico y financiero propuestos por el Ministerio de Desarrollo Internacional del Gobierno Británico (DFID), en el contexto de su participación en el Proyecto RUTA (Unidad Regional para Asistencia Técnica) y que han desarrollado el enfoque de medios de vida sostenibles desde el año 2001 (Cuadro 2).

La organización de la información por capitales, se logró por medio de una base de datos electrónica utilizando formularios y tablas que permitieron un acceso más fácil a la información recopilada de las fincas.

\section{Selección y validación de los indicadores de sostenibilidad en la fincas}

La selección y validación de los indicadores de la sostenibilidad se basó en el uso de indicadores propuestos por los siguientes estudios: la metodología MESMIS (Masera et al. 1999, López-Ridaura et al. 2001), el Sistema agroecológico rápido de evaluación de calidad de suelo y salud de cultivos en el agroecosistema de café (Altieri \& Nicholls 2002), Enfoque metodológico: emisión de gases 
CUADRO 2

Capitales, variables utilizados para la caracterización de la finca

\begin{tabular}{|c|c|c|}
\hline Capitales & Variables & Insumos- CEDECO \\
\hline Físico & $\begin{array}{l}\text { Red de caminos } \\
\text { públicos, alumbrado, } \\
\text { casas, bodegas, } \\
\text { caminos }\end{array}$ & $\begin{array}{l}\text { Mapas digitales } \\
\text { de la finca y de la } \\
\text { comunidad }\end{array}$ \\
\hline Natural & $\begin{array}{l}\text { Cultivos, abonos } \\
\text { orgánicos, manejo } \\
\text { de la finca, biomasa } \\
\text { microbiana, conteo } \\
\text { de artrópodos, } \\
\text { comunidades } \\
\text { vegetales, agro } \\
\text { biodiversidad }\end{array}$ & $\begin{array}{l}\text { Información } \\
\text { socioeconómica, } \\
\text { eficiencia energética, } \\
\text { carbono de suelos, } \\
\text { microbiología de } \\
\text { suelos }\end{array}$ \\
\hline Social & $\begin{array}{l}\text { Instituciones, } \\
\text { asociaciones y } \\
\text { organizaciones de } \\
\text { apoyo y participación }\end{array}$ & $\begin{array}{l}\text { Información } \\
\text { socioeconómica }\end{array}$ \\
\hline Humano & $\begin{array}{l}\text { Información familiar } \\
\text { y conocimientos } \\
\text { adquiridos }\end{array}$ & $\begin{array}{l}\text { Información } \\
\text { socioeconómica }\end{array}$ \\
\hline Financiero & $\begin{array}{l}\text { Eficiencia energética, } \\
\text { financiamiento, } \\
\text { productividad, mano } \\
\text { de obra }\end{array}$ & $\begin{array}{l}\text { Información } \\
\text { socioeconómica, } \\
\text { eficiencia energética }\end{array}$ \\
\hline
\end{tabular}

efecto invernadero, la fijación de carbono y la agricultura orgánica (Castro \& Amador 2006) y Metodología para evaluar sistemas campesinos de caficultores orgánicos desarrollada en Colombia (Cárdenas et al. 2005).

Para mejorar el análisis de sostenibilidad de las fincas por medios indicadores, este estudio propuso tres indicadores relacionados con servicios ecosistémicos (agrobiodiversidad, diversidad de arboles y conservación de bosques). En total se seleccionaron 15 indicadores para el análisis, los cuales fueron agrupados en siete atributos (Apéndice 1).

Cada indicador fue valorado mediante una escala de 1 a 3 , donde 1 fue el valor más bajo y tres el valor más alto. Posteriormente, se obtuvo el promedio al sumar la totalidad de los indicadores por finca, lo que permitió establecer el índice de sostenibilidad. Además, se estableció un umbral de sostenibilidad a partir del valor de 2 (valor mínimo de una magnitud a partir de la cual se produce un efecto determinado) donde valores mayores a 2 se consideran que tienden a la sostenibilidad y valores menores a 2 se consideran por debajo del umbral y por tanto, no son sostenibles.

\section{RESULTADOS}

\section{Caracterización del sistema agrícola de las fincas por cultivo}

El Apéndice 2 presenta una sinopsis de la caracterización de los sistemas agrícolas ecológico y tradicional por cultivo de las fincas en estudio. La caracterización se realizó en base a los capitales humano, social, financiero, natural y físico, recopilados en la base de datos electrónica.

\section{Sostenibilidad}

Los índices obtenidos muestran que las fincas ecológicas presentan niveles por encima del umbral, alcanzando una tendencia hacia la sostenibilidad dentro del rango establecido en esta investigación. En el caso de las fincas tradicionales, se encuentran por debajo del umbral, por lo tanto, no tienden a ser sostenibles (Fig. 2). En cuanto a los indicadores las fincas ecológicas con respecto a las tradicionales, muestran fortalezas en el manejo de suelo mediante la implementación de prácticas de conservación, muestran un mayor aporte en servicios ecosistémicos y una alta productividad similar a fincas tradicionales, esto hace que las fincas ecológicas presenten tendencia hacia la sostenibilidad (Fig. 3).

\section{DISCUSIÓN}

La metodología diseñada para este estudio permitió analizar que las fincas con manejo ecológico tienden a ser sostenibles en aspectos relacionados con eficiencia energética, manejo del suelo, provisión de servicios ecosistémicos y aspectos sociales. Igual resultado se han obtenido en estudios metodológicos realizados en el 2007 (AriasGiraldo \& Camargo 2007, Cárdenas et al. 2007) donde los sistemas agrícolas ecológicos presentaron niveles altos en los indicadores establecidos en la parte ambiental, social y económica.

La caracterización de las fincas, permitió apreciar que el sistema de manejo ecológico presente en 10 de las 17 fincas, esta representado de forma general por ser unidades de manejo agrobiodiversos, con reciclaje de nutrientes, con grupos familiares pequeños y con alta productividad. Resultados similares se obtuvo en dos fincas de la comunidad de San Antonio de los Baños, Habana, Cuba (Monzote et al. 2008) como parte de un seguimiento del 2000 al 2004 de indicadores de sostenibilidad relacionados con la parte biológica de los sistemas, productividad, eficiencia energética y economía de fincas ecológicas. 


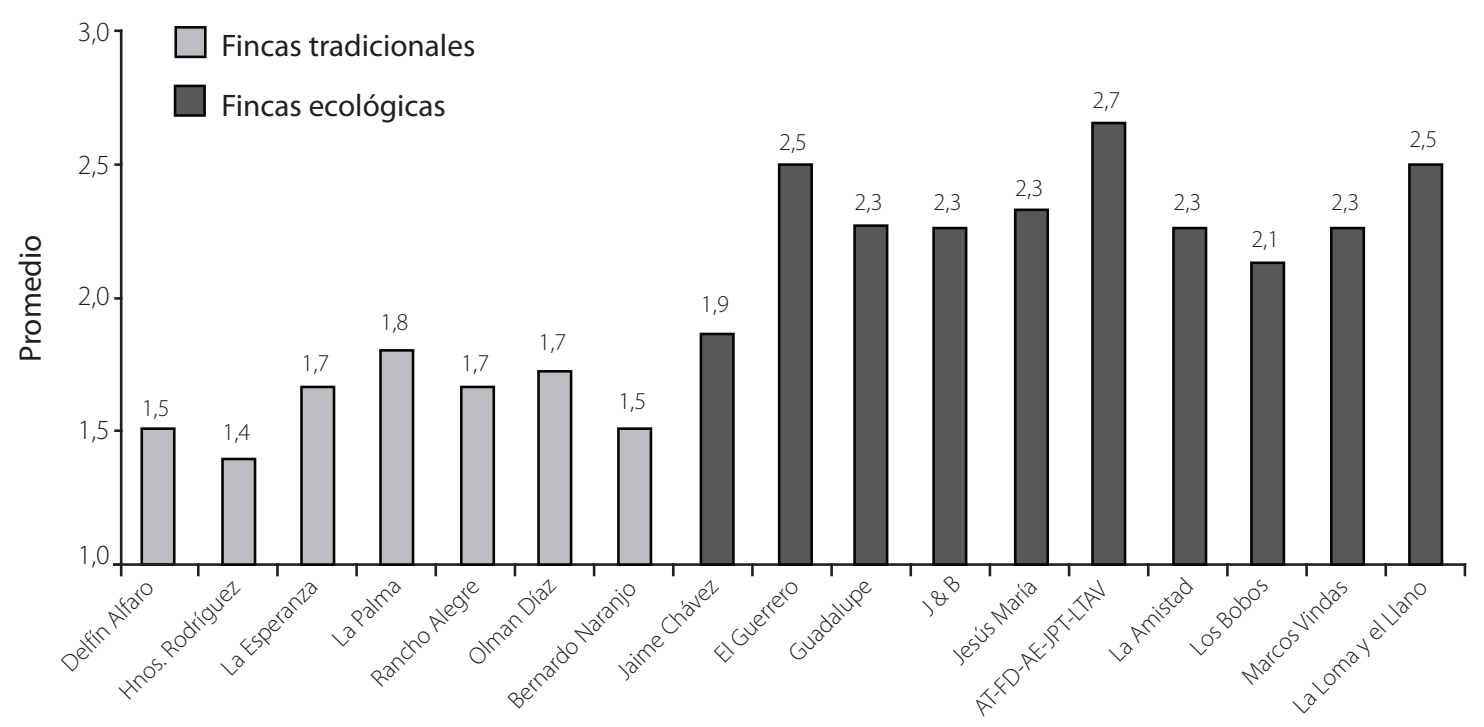

Fincas

FIG. 2. Índice de sostenibilidad para cada finca según el promedio obtenido en la valoración de los indicadores.
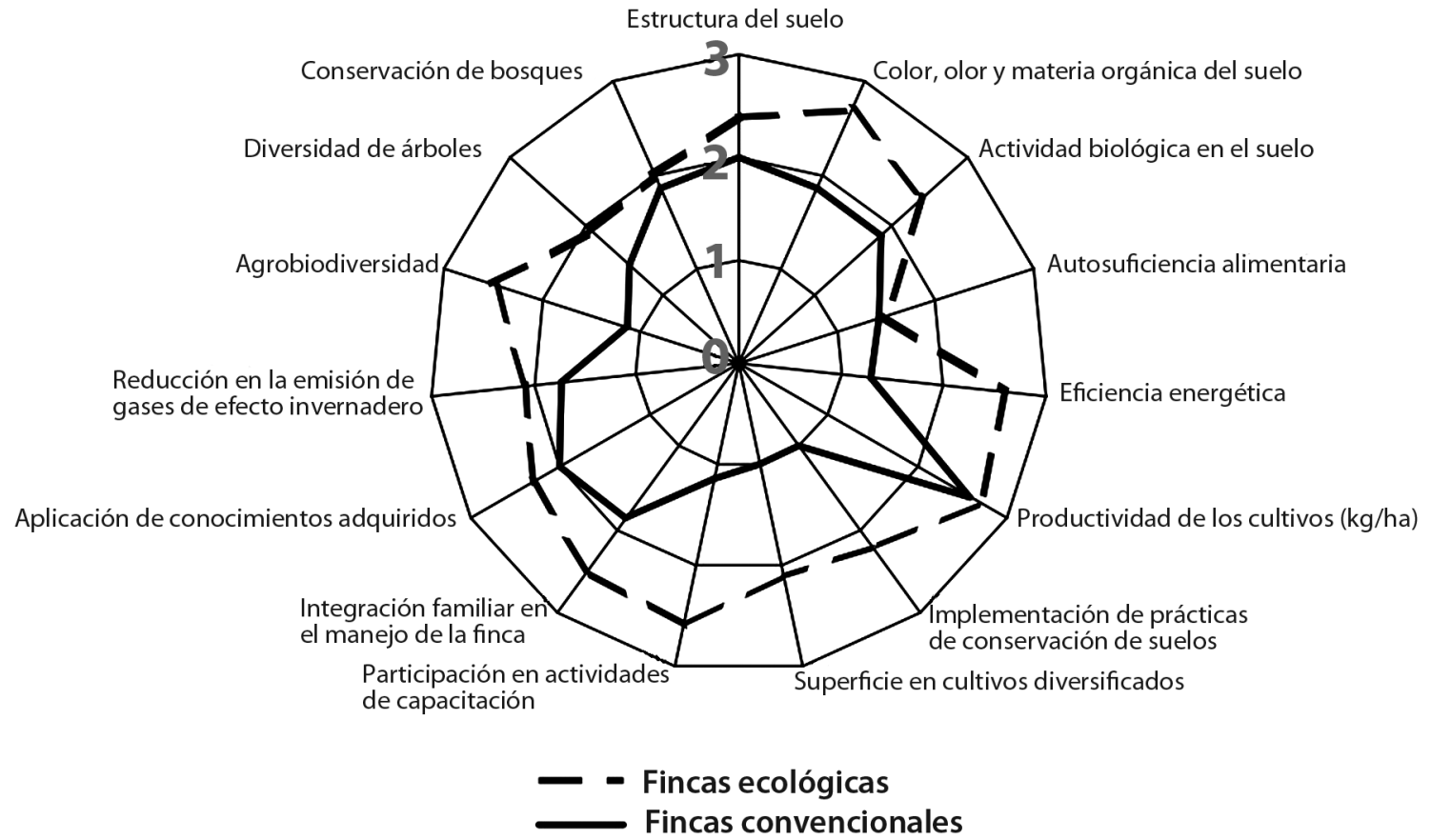

FIG. 3. Diagrama de sostenibilidad de los valores generales para las fincas ecológicas y tradicionales. 
En fincas con manejo tradicional, el agroecosistema está basado en el empleo del monocultivo como medio de producción, aplicación de insumos químicos, un pequeño grupo familiar y alta productividad. Además, poseen suelos con problemas de erosión y poca presencia de materia orgánica, lo que causa un incremento de insumos químicos para hacer el suelo fértil y así obtener niveles de productividad altos, agravando el medio biofísico.

Los atributos empleados en esta investigación para la valoración de los indicadores mostraron que en productividad, adaptabilidad y estabilidad hay una correlación implícita en los sistemas ecológicos. En las fincas ecológicas los suelos presentan abundante materia orgánica, una estructura estable y micro fauna abundante, que aunado a prácticas de conservación en el suelo, le dan una mayor fertilidad, manteniendo niveles de productividad altos y permitiendo mayores ganancias económicas sin invertir en gastos como insumos y fertilizantes químicos. En las fincas tradicionales la adaptabilidad y estabilidad resultaron con valores bajos, no así la productividad mostrando valores altos, sin embargo son sistemas con baja materia orgánica producto de los problemas de erosión y poca aplicación de prácticas de conservación de suelos, el nivel de productividad esta ligado a un empleo de insumos químicos y a la producción de un solo cultivo.

Los indicadores para el análisis y valoración de las fincas en la investigación, resultaron similares con los establecidos en la metodología del MESMIS (Masera 1999), en lo que se refiere a los requisitos que deben cumplir para ser utilizados, es decir, que dieron información condensada sobre distintos atributos, fueron fáciles de medir, sencillos, claros y concentrados en aspectos del sistema agrícola a analizar.

Los resultados obtenidos con los indicadores de la metodología del MESMIS en los diferentes estudios que se han realizado (Masera et al. 2000, Astier et al. 2004, Monterroso 2004, Gomero \& Velásquez 2004, Cárdenas et al. 2007), evidencian que los sistemas ecológicos tienden a ser sostenibles debido principalmente a que presentaron valores altos en los indicadores en los atributos de adaptabilidad, estabilidad, organización, eficiencia y productividad. En esta investigación los resultados son similares, en cuanto a los atributos, sin embargo, el atributo de servicios de los ecosistemas, es novedoso y aportó nuevos fundamentos científicos para demostrar la sostenibilidad de sistemas agrícolas, además permitió darle más énfasis al aporte a la conservación de la biodiversidad local, el manejo de semilla criolla y reducción de gases de cambio climático.

El empleo de atributos e indicadores para analizar fincas son elementos útiles y sencillos, los cuales pueden ser usados por el agricultor o productor, asesores técnicos, organizaciones e instituciones, para proporcionar una visión general del comportamiento de la finca y evaluar el impacto que sus actividades generan al ambiente.

Queda demostrado que las fincas con manejo ecológico tienden hacia la sostenibilidad debido a que llevan a cabo prácticas de conservación de suelo, reciclan sus nutrientes, conservación biodiversidad, mejoramiento del paisaje y hay un mayor involucramiento de la familia en las labores de la finca y en la comunidad.

La metodología desarrollada por esta investigación contribuirá en estudios sobre el pago de servicios ecosistémicos a fincas ecológicas, debido al papel de las fincas en la conservación de la biodiversidad local, conectividad de áreas boscosas y aporte en la reducción del cambio climático.

\section{AGRADECIMIENTOS}

A las familias propietarias de las fincas analizadas, por su colaboración en la toma de datos y por abrir sus conocimientos al sector productivo y agrícola del país. A CEDECO por su aporte científico y logístico en la realización de esta investigación.

\section{REFERENCIAS}

Altieri, M. \& C. Nicholls. 2000. Agroecología: teoría y práctica para una agricultura sostenible. PNUMA, D.F. México.

Altieri, M. \& C. Nicholls. 2002. Sistema agroecológico rápido de evaluación de calidad de suelo y salud de cultivos en el agroecosistema de café. (http://www.agroeco.org/doc/ SisAgroEvalSuelo2.htm; consultado 11 de mayo, 2008).

Arias-Giraldo, M. \& C. Camargo. 2007. Análisis de Sostenibilidad en unidades productivas ganaderas del municipio de Circasia (Quindío - Colombia), Cuenca del Río La Vieja. (http://www.cipav.org.co/lrrd//rrd19/10/aria19149.htm; consultado 25 de agosto, 2007).

Astier, M. \& O. Masera. 1997. Metodología para la evaluación de sistemas de manejo incorporando indicadores de sostenibilidad (MESMIS). Mundi-Prensa GIRA, Patzcuaro, México.

Astier, M., A. Peréz, T. Ortiz \& F. Mota. 2004. Sostenibilidad de sistemas campesinos de maíz después de cinco años: el segundo ciclo de evaluación MESMIS. Revista Leisa de Agroecología 20: 39-47.

Bifani, P. 1995. El desafío ambiental como un reto a los valores de la sociedad contemporanea, Master en Educación Ambiental, Fundación Universidad-Empresa, Madrid, España.

Cárdenas, G., H. Giraldo, A. Idárraga \& L. Vásquez . 2007. Desarrollo y Validación de Metodología para Evaluar con 
Indicadores la Sostenibilidad de Sistemas Productivos Campesinos de la Asociación de Caficultores Orgánicos de Colombia-ACOC. (http://www.javeriana.edu.co/fear/ m_des_rur/documents/Cardenas-presentacion.pdf; consultado 26 de agosto, 2008).

Castro, J. \& M. Amador. 2006. Enfoque metodológico: emisión de gases efecto invernadero, la fijación de carbono y la agricultura orgánica. Aportes para la Educación. San José, Costa Rica.

Food and Agriculture Organization (FAO). 2007. El Estado Mundial de la Agricultura y la Alimentación. Subdirección de Políticas y Apoyo en Materia de Publicación Electrónica. Dirección de Comunicación. Roma, Italia.

Gomero, O. \& H. Velásquez. 2004. Evaluación de la sostenibilidad del sistema de algodón orgánico en la zona de trópico húmedo de Perú. Revista Leisa de Agroecología 20: 4757.

IPCC. 2007. Cambio climático 2007. Informe de síntesis. Contribución de los Grupos de trabajo I, II y III al Cuarto Informe de evaluación. Grupo Intergubernamental de Expertos sobre el Cambio Climático, Ginebra, Suiza.

Leff, E. 1996. La Capitalización de la Naturaleza y las Estrategias Fatales de la Sostenibilidad. Revista Formación Ambiental 16: 17-20.

López-Ridaura, S., O. Masera \& M. Astier. 2001. Evaluando la sostenibilidad de los sistemas agrícolas integrados: El mar- co MESMIS. Revista Leisa de Agroecología 16: 25- 27.

Masera, O. \& S. López-Ridaura. 2000. Sostenibilidad y Sistemas Campesinos. Cinco experiencias de evaluación en el México rural. MundiPrensa-GIRA, UNAM, México.

Masera, O., M. Astier \& S. López-Ridaura. 1999 Sostenibilidad y manejo de recursos naturales: el marco de evaluacion MESMIS. Mundiprensa, GIRA, UNAM, México.

Monterroso, N. 2004. Evaluación social de la sostenibilidad de dos comunidades rurales del Estado de México. Red Internacional para el Manejo Sostenible de los Recursos Naturales, Toluca, México.

Mooney, P. 1993. Structure and connectivity as measures of sustainability in agroecosystems. In J. Marczyk \& D. Johnson (eds.). Sustainable Landscape, Proceedings of the Third Symposium of CSLEM, Alberta, June 1992. Polyscience, Marin Heights, Canada, p. 13-25.

Monzote, F., A. Hernández, R. Bello \& A. Alvárez. 2008. Fertilidad del suelo a largo plazo en sistemas biointensivos. Revista Leisa de Agroecología 24: 9-12.

WRI (Instituto Mundial sobre Recursos). 2005. World Resources 2005: The wealth of the poor-Managing ecosystems to fight poverty. En colaboración con PNUD, PNUMA y Banco Mundial. Washington, DC, EEUU. 


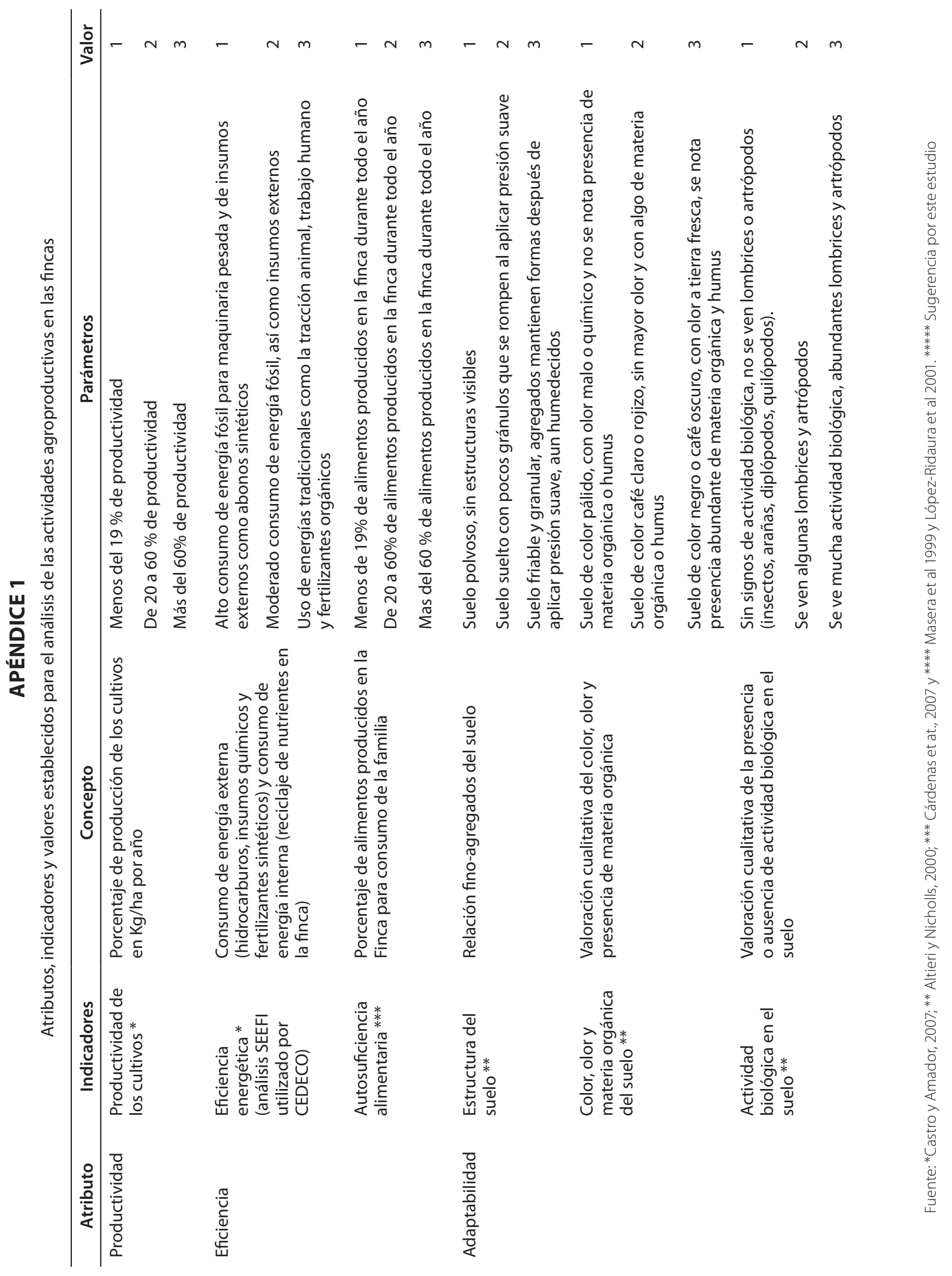




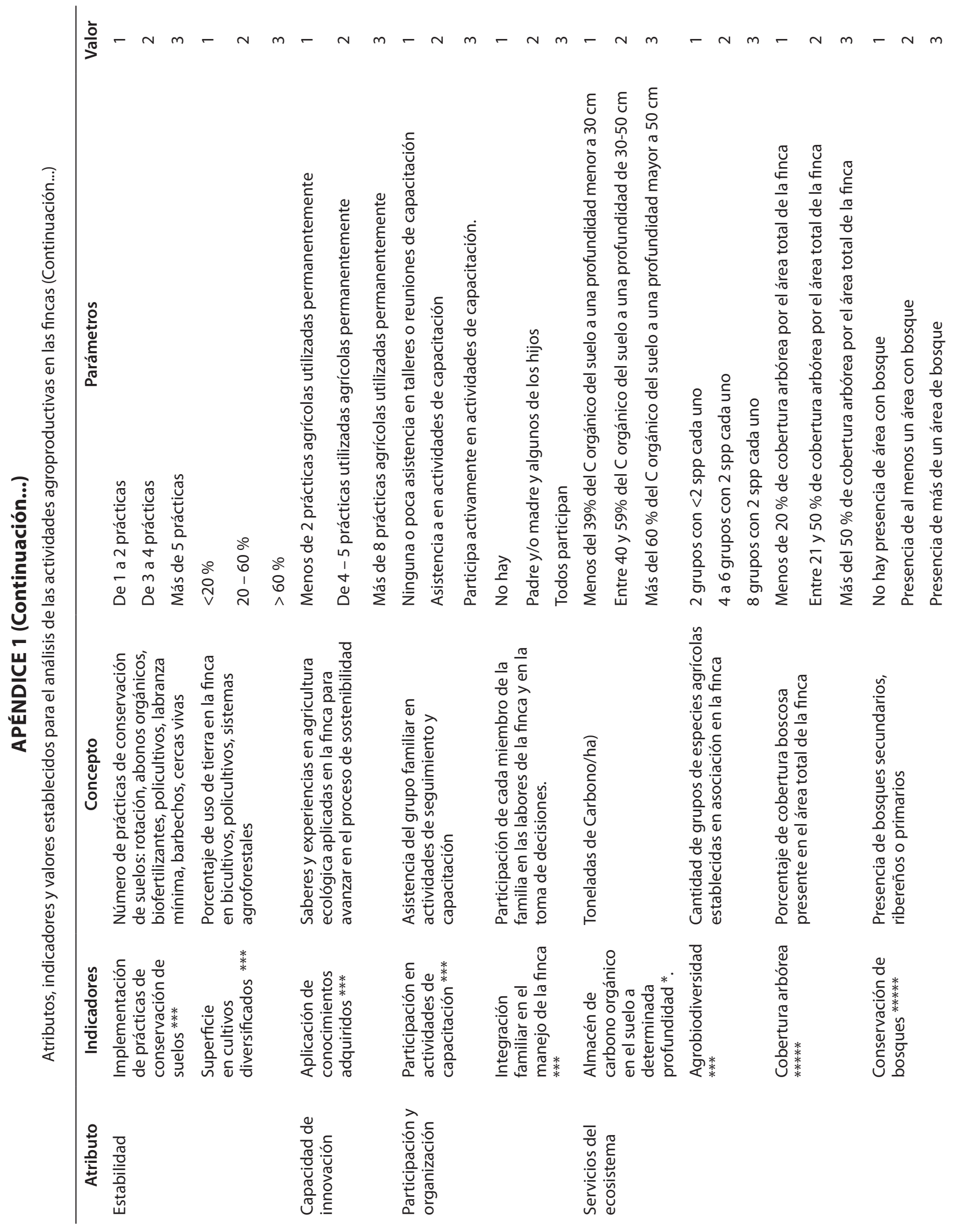




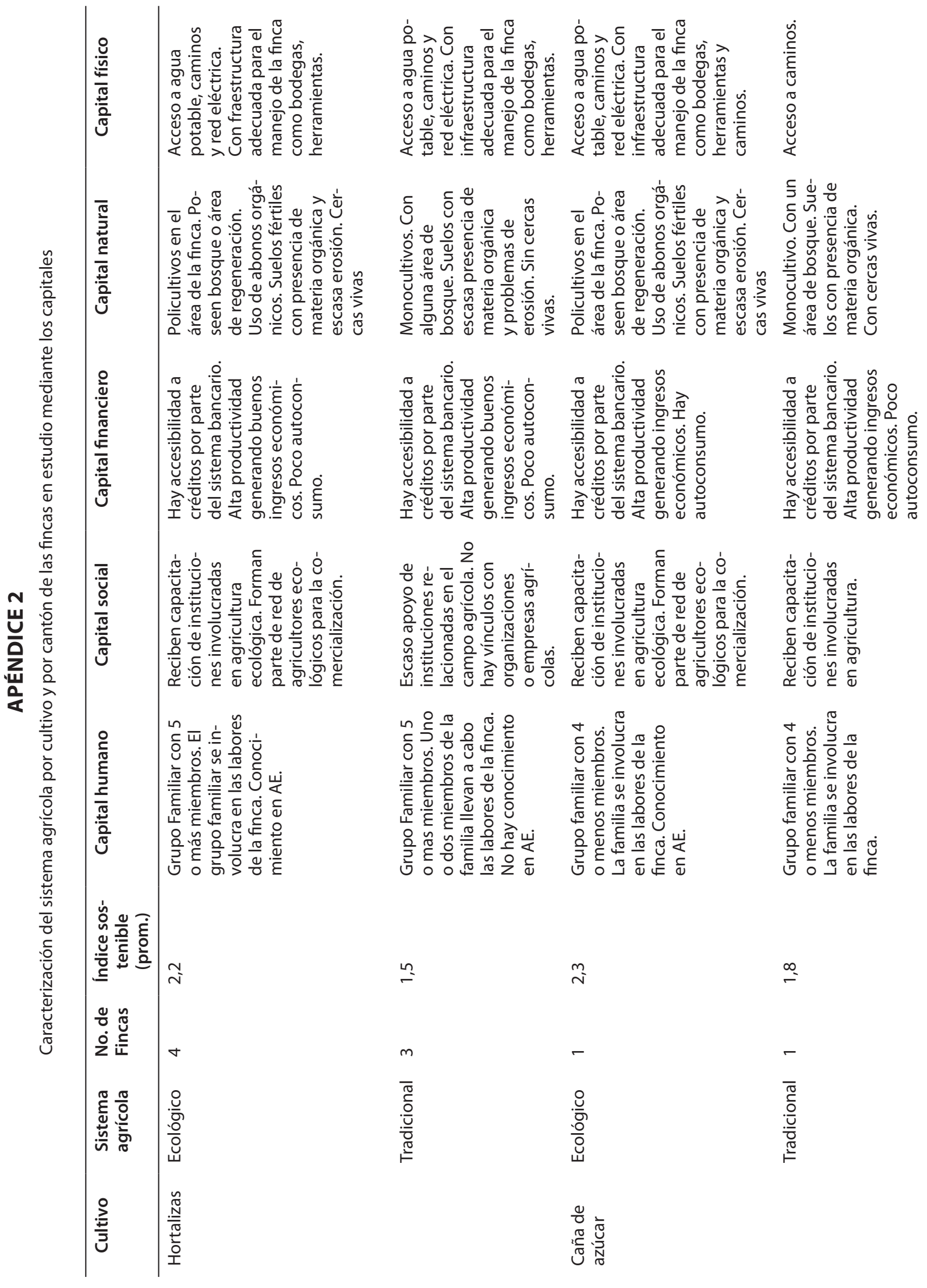



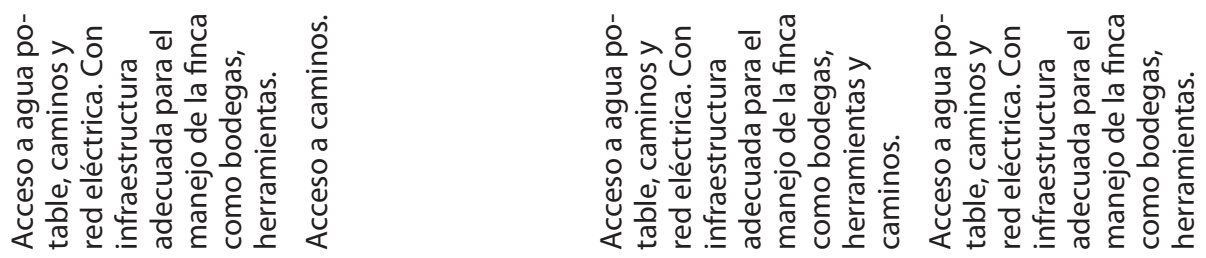

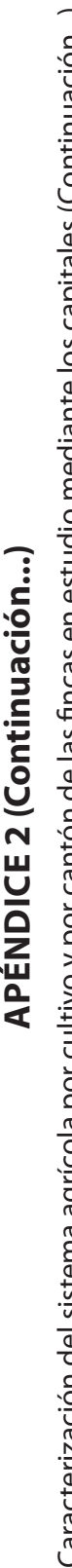
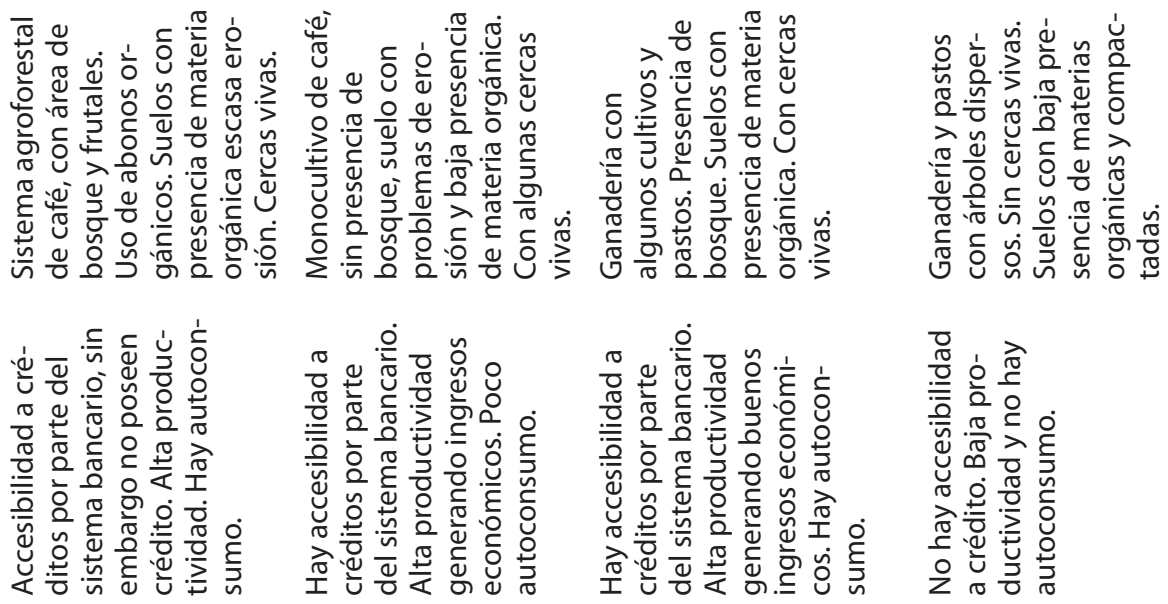

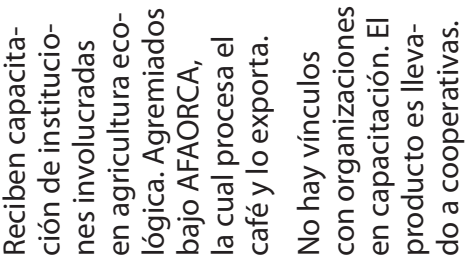
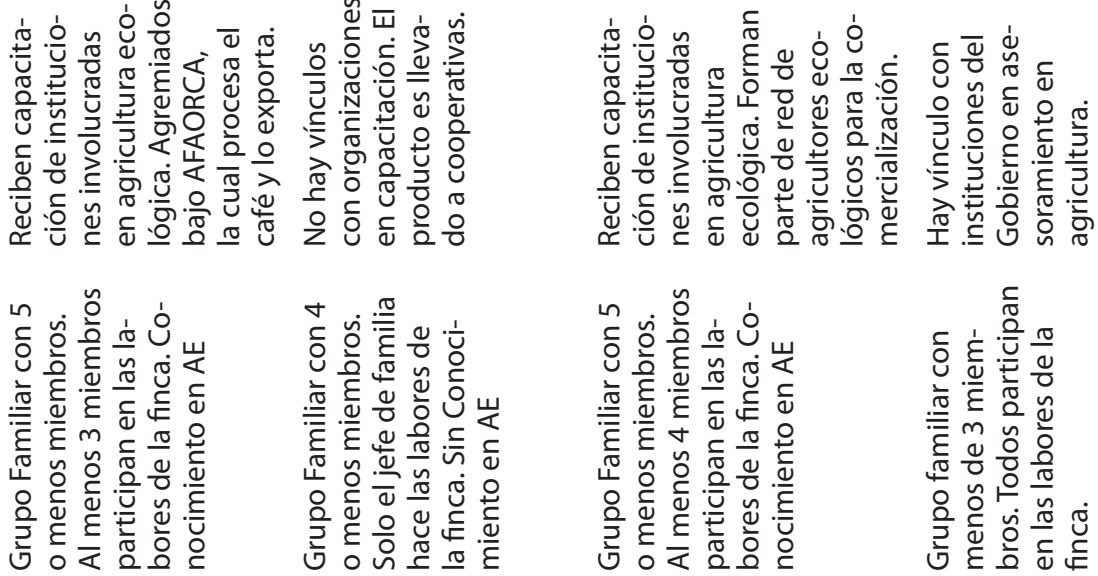

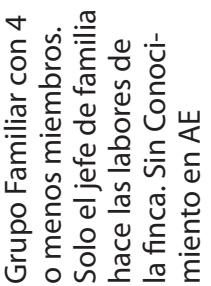
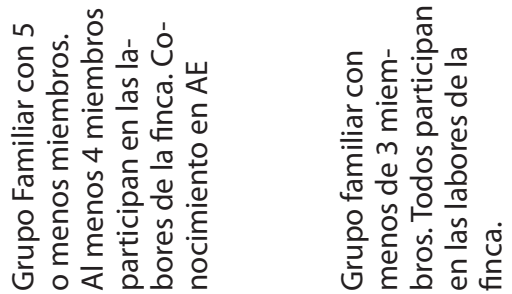

n

$\stackrel{\bullet}{\sim}$

$\stackrel{n}{N}$

气

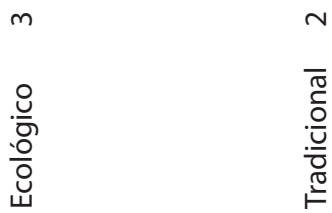

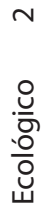

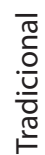

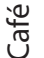

> 